Journal of Sol-Gel Science and Technology 34, 211-217, 2005
(c) 2005 Springer Science + Business Media, Inc. Manufactured in The Netherlands.

\title{
Stability of Suspensions of Bioactive Particles Using Hybrid Organic-Inorganic Solutions as Dispersing Media
}

\author{
C. GARCIA* \\ Universidad Nacional de Colombia Sede Medellín, Facultad de Ciencias, Escuela de Física, \\ A.A. 3840 Medellín, Colombia \\ cpgarcia@unalmed.edu.co \\ A. DURÁN AND R. MORENO \\ Instituto de Cerámica y Vidrio (CSIC), Campus de Cantoblanco, 28049 Madrid, Spain
}

Received June 13, 2003; Accepted August 25, 2004

\begin{abstract}
Functional coatings incorporating different types of particles developed by the sol-gel method have been proposed in the last few years for diverse applications. This work focuses on the preparation of homogeneous coatings prepared from stable suspensions with $10 \mathrm{wt} \%$ of glass and glass ceramic particles in a hybrid organic-inorganic solution as dispersing media. For this purpose, the $\mathrm{pH}$ was shifted up to 6-7 by adding tetrapropylammonium hydroxide (TPAH) which behaves as a cationic surfactant being probably adsorbed on the particles surface, while the sol maintains stable. Rheological measurements were performed to study the stability of the suspensions prepared at different conditions such as the kind and concentration of dispersant and the $\mathrm{pH}$ conditions. After sintering at $450^{\circ} \mathrm{C} / 30 \mathrm{~min}$, coatings around $2 \mu \mathrm{m}$ in thickness were obtained.
\end{abstract}

Keywords: rheology, bioactive particles, coatings, sol-gel

\section{Introduction}

The manufacture of sol-gel derived coatings is a promising application of sol-gel technology, since a variety of coating materials can be applied on various substrates, such as metals, glasses and ceramics, and without expensive equipment. Furthermore, these coatings allow for improvement in the mechanical, thermal, optical, protective, bioactive and electrical properties of the substrate material [1-8].

The most widely used method for obtaining sol-gel coatings is dipping, in which the substrate is immersed in the solution and withdrawn at a controlled rate. However, the coatings obtained by single-dipping have a

*To whom all correspondence should be addressed. critical thickness, defined as the maximum thickness without cracks, usually less than $2 \mu \mathrm{m}$.

The corrosion resistance and the protective character of the coatings could increase as it becomes thicker. Some authors have developed functional coatings through the sol-gel method by incorporating glass and ceramic particles [5, 9]. These works remark the importance of preparing stable suspensions of particles in the precursor solution, if defect-free coatings are to be obtained.

Much effort has been devoted to study the mechanisms responsible for the dispersion of particles in a medium. However, current knowledge mainly refers to the dispersion of particles in a pure solvent (or a mixture of solvents), where stability can arise from either the formation of a double layer around the 
particles that promotes electrostatic repulsion, or the steric repulsion obtained by adsorbed molecules on the surface.

Another possibility, which has received less attention in the literature, is the dispersion of particles in a precursor sol-gel solution, instead of a solvent. In this case, the interactions are much more complex, since the precursor solution consists of a hybrid organic-inorganic solution formed by alkoxide chains which continuously evolve to form a structural network. This means that the stability of the solution must be ensured before the particles are added. In a second step, care must be taken to obtain stability and a uniform distribution of particles added to the solution. Hence, rheological measurements are a powerful tool for studying the preparation conditions of sol-gel suspensions and for improving the homogeneity of the coatings.

Previous work [9] reported the preparation of coatings from suspensions of glass with average particle size of $5 \mu \mathrm{m}$ and solid contents up to $25 \%$, concluding that a content of particles in the suspension higher than $10 \mathrm{wt} \%$ led to lines of drainage in the coatings. That work focused on the in vitro evaluation of coatings concluding that a two stage sol-gel coating system that included a first $\mathrm{SiO}_{2}$ hybrid film, acting as a barrier against corrosion, and an external bioactive layer, built up from glass particle suspension, is a good alternative to improve the properties of stainless steel $316 \mathrm{~L}$.

The present work focuses on the preparation of homogeneous and dense sol-gel bioactive coatings with glass and glass ceramic particles. Our final goal is to manufacture well-controlled, reliable coatings containing bioactive particles by the sol-gel route and to determine the parameters that influence over the process, facing the future development of bioactive coatings on metallic biomedical substrates. Some properties of this kind of substrates, such as corrosion resistance and bioactivity, should be improved. For this purpose, stable suspensions of bioactive glass and glass-ceramic particles using hybrid organic-inorganic solutions as dispersing media have been prepared and rheologically characterized.

In the present work coarser particles (average diameter $20 \mu \mathrm{m}$ ) have been used, thus requiring a new optimization of the dispersing conditions capable to stabilize both the glass and the glass-ceramic coarse particles. The concentration of particles has been fixed to $10 \mathrm{wt} \%$ in order to avoid drainage lines in the coatings.

\section{Experimental}

The first step required in preparation of bioactive coatings by the sol-gel method is production of a stable suspension of glass or glass-ceramic particles dispersed in a sol. The suspensions thus prepared are then deposited onto a substrate and thermally treated.

\subsection{Glass Melting and Milling}

Glass in the system $\mathrm{CaO}-\mathrm{SiO}_{2}-\mathrm{P}_{2} \mathrm{O}_{5}$ with composition $57.44 \% \mathrm{CaO}, 35.42 \% \mathrm{SiO}_{2}$ and $7.15 \% \mathrm{P}_{2} \mathrm{O}_{5}$ in molar percentages was obtained by melting using reactive grade chemicals: calcium carbonate (Aldrich), orthophosphoric acid (Aldrich), and purified silica sand. The batch was melted at $1600^{\circ} \mathrm{C}$ for $2.5 \mathrm{~h}$ and quenched in water at room temperature. This glass presents the formation of apatitic phase films on the surface of the coatings in a simulated body fluid, as a result of the chemical reaction between the glass and the surrounding body fluid, considered as a clear sign of bioactivity [9-12].

Glass-ceramic particles were obtained from the previously described fritted glass, through heat treatment for $2 \mathrm{~h}$ at $1050^{\circ} \mathrm{C}$, yielding partially crystallised powders.

The vitreous or crystalline character of the resulting particles was evaluated by X-ray diffraction, using a Siemens Difrac 5000.

Both the glass and the glass-ceramic particles were milled in a planetary mill (Fritsch Pulverisette, Germany) with agate jar and balls, using a speed of rotation around $1500 \mathrm{rpm}$ for a maximum period of $7 \mathrm{~h}$.

After milling, the particle size distribution was measured using laser diffraction analyzer (Mastersizer S, Malvern, U.K.). The specific surface area was determined using a $\mathrm{N}_{2}$-adsorption BET (Monosorb, Quantachrome, USA).

\subsection{Preparation of the Sols}

The sols were prepared by the method of acid catalysis in a single stage. Tetraethyl orthosilicate (TEOS, $\mathrm{ABCR}$ ) and methyltriethoxyisilane (MTEOS, ABCR) were selected as silica precursors for the sol, prepared in alcoholic media using $\mathrm{HNO}_{3} 0.1 \mathrm{~N}$ (acidulated water) and acetic acid as catalysts. In order to obtain a final concentration of $\mathrm{SiO}_{2}$ of $200 \mathrm{~g} / \mathrm{l}$, the parameters were chosen as follow: The molar ratio TEOS:MTEOS was 
fixed as 40:60, the $\mathrm{H}_{2} \mathrm{O}$ :(TEOS + MTEOS) ratio was equal to $2: 1$ and the water:acetic acid ratio was $7: 1$.

The solutions were prepared by mixing absolute ethanol with the alkoxides at room temperature and maintained in agitation with a magnetic stirrer. The acetic acid and the acidulated water were added drop by drop to catalize the hydrolysis and condensation reactions. After 10 min of stirring, the beaker was transferred to a thermostatic bath and the solution was maintained at $40^{\circ} \mathrm{C}$ and constantly stirred for three hours until a transparent and limpid sol was obtained with a $\mathrm{pH}$ of 1.0 to 2.0 .

The stability of the as-prepared sol ( $\mathrm{pH} 1-2)$ was evaluated by measuring the viscosity and its variation with time. Rheological measurements were performed using a rotational rheometer (Haake RS50, Germany) with a double-cone and plate measuring system. The stability of the sol as a function of $\mathrm{pH}$ was evaluated by adding tetrapropylammonium hydroxide (TPAH, ALDRICH) to the previously prepared sol up to $\mathrm{pH}$ values of 6 to 7 . The effect of TPAH addition on the viscosity of the sol with elapsed time was also determined.

\subsection{Preparation of the Suspensions}

The suspensions were prepared to a solid loading of $10 \mathrm{wt} \%$ by homogenising with a high shear mixer (Silverson L2R, UK) for $3 \mathrm{~min}$. Later, the required amount of TPAH was added and the suspensions were further stirred for $3 \mathrm{~min}$. The stability of the suspensions was studied by measuring the flow curves for different concentrations of TPAH and by qualitative examination of the appearance of the suspension and the tendency to settle. The optimum concentration of TPAH was determined from the deflocculation curve, where the viscosity is plotted as a function of the TPAH content at a constant value of shear rate. Once the adequate amount of TPAH was established, the stability of the suspensions with respect to time was studied.

The surface charge of the particles was determined by electrophoresis using a power supplier (AMEL 551, UK) and graphite electrodes. Tests were performed applying a potential of $11 \mathrm{~V}$ for a deposition time of $3 \mathrm{~min}$. The mass per unit area was calculated by weighting the electrodes before and after the tests.

\subsection{Preparation and Characterization of the Coatings}

Microscope glass slides of $7.5 \mathrm{~cm} \times 2.5 \mathrm{~cm}$ were used as substrates. Coatings were prepared by dip coating with equipment that allows for control of the withdrawal rate between 4 and $65 \mathrm{~cm} / \mathrm{min}$. Coatings were produced at several withdrawal rates to determine the critical thickness of each system. The coatings were dried at room temperature and heat treated at $450^{\circ} \mathrm{C}$ for $30 \mathrm{~min}$. The thickness was measured with a profilometer (Talystep, Taylor Hobson, USA) on a scratch marked on the film after the deposition. The integrity and characteristics of the coatings were evaluated by optical microscopy (Olympus BX41, USA) and the images were analysed using appropriate software (Lyca, USA).

\section{Results and Discussion}

\subsection{Characterization of the Glass and Glass-Ceramic Particles}

The glass particles are colourless and transparent and do not contain crystalline phases, as confirmed in the XRD diffractogram shown in Fig. 1(a). The glassceramic particles are white and contain well defined crystalline phases identified as hydroxyapatite and wollastonite, Fig. 1(b).

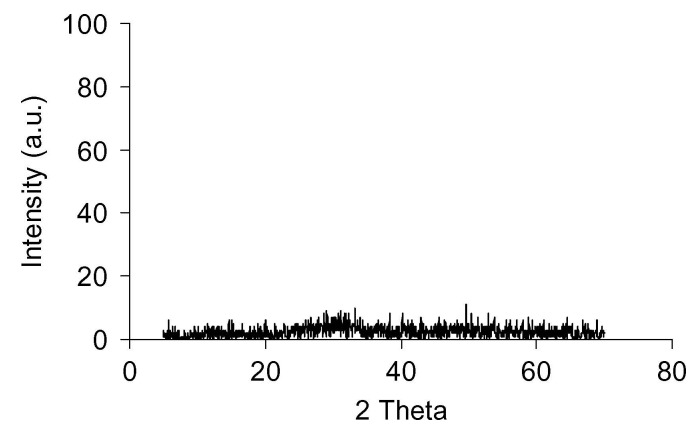

(a)

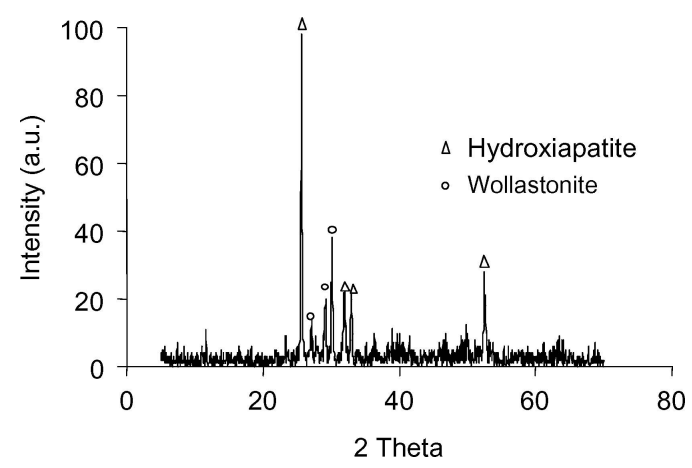

(b)

Figure 1. X-ray diffraction patterns of the particles: (a) Glass and (b) Glass-ceramics. 
Table 1. Mean size, density and surface area of the milled particles. The error bars was calculated from a statistical propagation of error analysis. Each parameter were measured at least two times for each sample, with four scans for each measurement.

\begin{tabular}{lccc}
\hline Particles & $\begin{array}{c}\text { Surface area } \\
\left(\mathrm{m}^{2} / \mathrm{g}\right)\end{array}$ & $\begin{array}{c}\text { Average diameter } \\
(\mu \mathrm{m})\end{array}$ & $\begin{array}{c}\text { Density } \\
\left(\mathrm{g} / \mathrm{cm}^{3}\right)\end{array}$ \\
\hline Glass & $0.3 \pm 0.1$ & $39 \pm 1$ & $2.92 \pm 0.05$ \\
Glass-ceramic & $0.8 \pm 0.1$ & $16 \pm 1$ & $2.99 \pm 0.05$
\end{tabular}

The average size and the specific surface area of the particles after milling are shown in Table 1 . These parameters were measured at least two times for each sample, with four scans for each measurement. The particle size distribution of the glass particles is narrow and centered around $40 \mu \mathrm{m}$, while that of the glassceramic particles is broader but the average diameter is finer $(16 \mu \mathrm{m})$.

\subsection{Characterization of the Starting Sol}

Figure 2 shows the evolution of viscosity with storage time of the precursor solution prepared at acidic conditions and that modified with TPAH. The as-prepared acidic sol follows Newtonian behaviour and remains stable with time for at least $350 \mathrm{~h}$, the viscosity increasing from $2.4 \mathrm{mPa} . \mathrm{s}$ to $3.8 \mathrm{mPa} . \mathrm{s}$ over $50 \mathrm{~h}$. On the other hand, the basic sol has a starting viscosity of $3 \mathrm{mPa} . \mathrm{s}$, which stays constant for $200 \mathrm{~h}$ and slightly increases to $4.2 \mathrm{mPa}$.s after $380 \mathrm{~h}$. Thus, the addition of TPAH is useful for preserving the stability of the sol for the first $200 \mathrm{~h}$.

\subsection{Characterization of the Suspensions}

Previous work reported the preparation of stable suspensions with glass particles using a phosphate ester as a dispersant [9]. In the present work, it was not possible to obtain stable suspensions with this dispersant $(\mathrm{pH}=$ $2-3$ ), and sedimentation occurred in a few minutes, as a result of the larger particle size.

In order to study the stability of the suspensions prepared with glass and glass-ceramic particles, the viscosities were measured for different additions of TPAH. The rheological behaviour of the suspensions and visual inspection give an idea of their stability and allows one to detect whether sedimentation occurs. The flow curves of the differently prepared suspensions can be observed in Figs. 3 and 4, for glass and glass-ceramic particles, respectively.

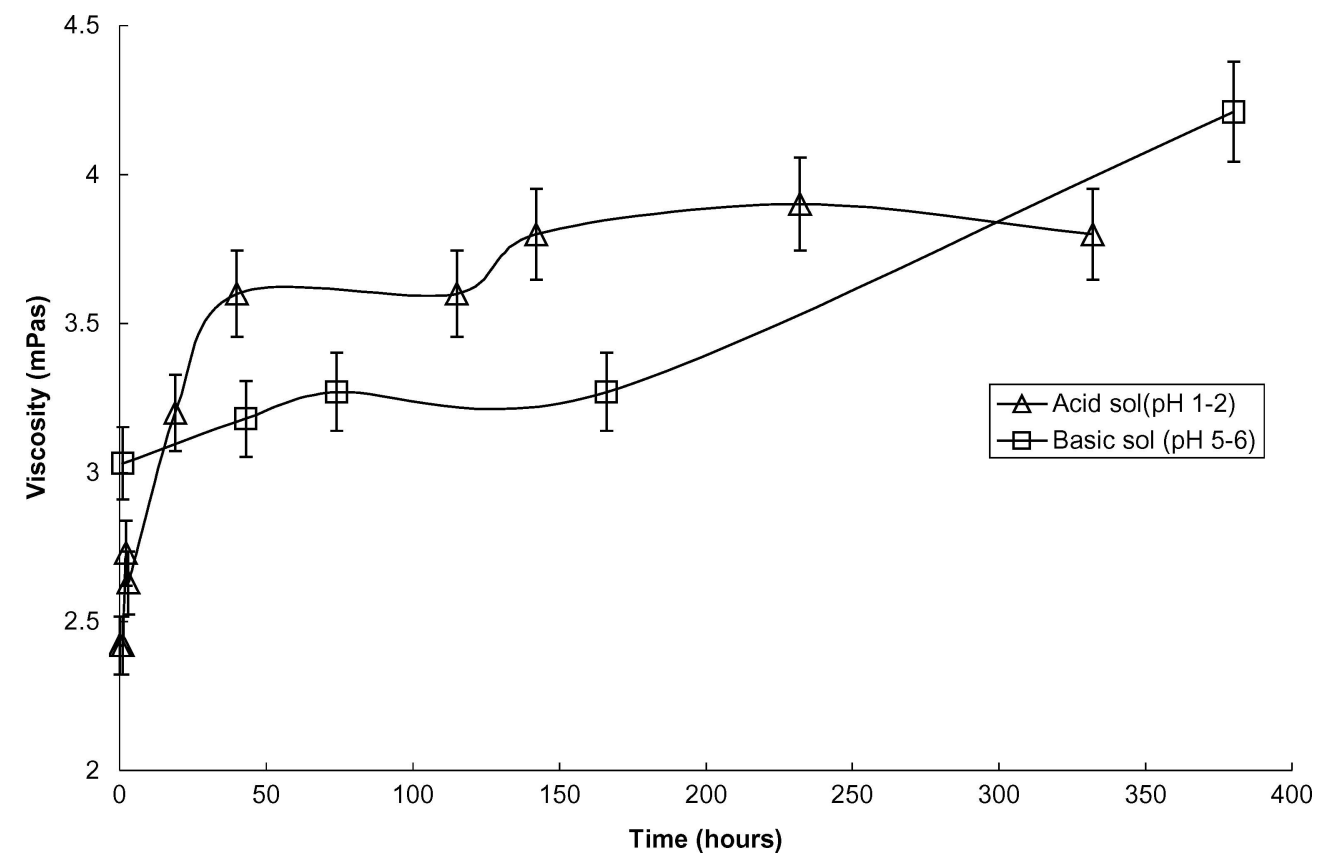

Figure 2. Variation of the viscosity with time for acid and basic sols. The errors in rheological measurements are given by the torque resolution, which depends on the shearing conditions. The maximum error of the measurements is below $4 \%$, but in practice, differences in rheometric determinations are mostly due to differences in the sample (i.e., shear history, sedimentation, formation of structure, etc). 


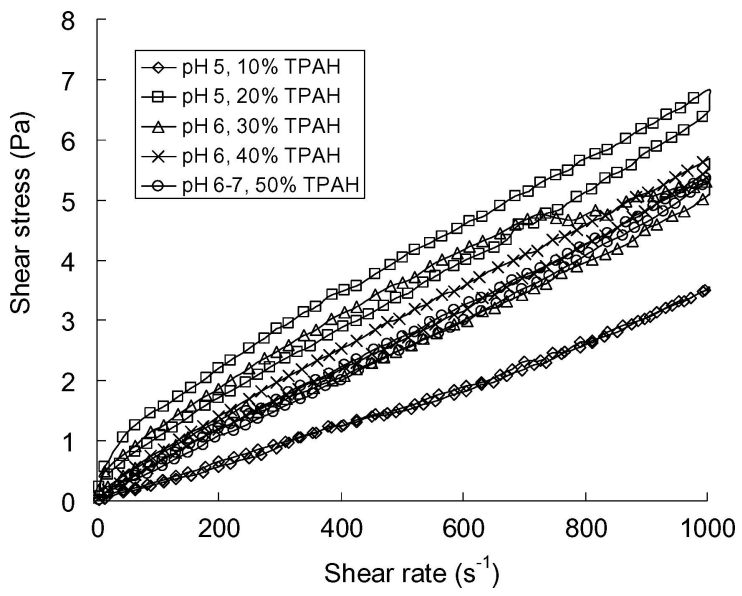

Figure 3. Flow curves $(\eta=\tau / \dot{\gamma})$ of suspensions of glass particles with the addition of TPAH (wt \%).

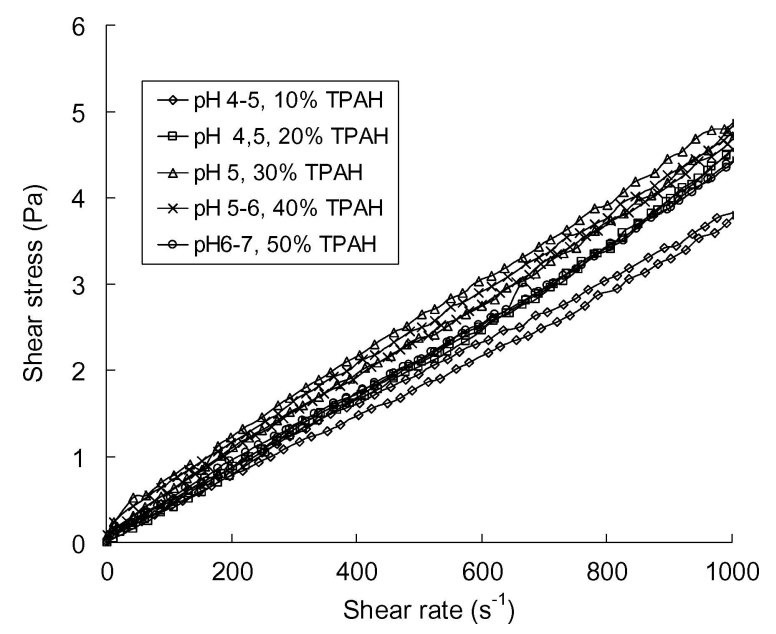

Figure 4. Flow curves $(\eta=\tau / \dot{\gamma})$ of suspensions of glass-ceramic particles with the addition of TPAH (wt $\%$ ).

The suspensions of glass and glass-ceramic powders prepared at acidic conditions were very unstable and settled in a few minutes. This is probably due to the large particle size and may also be related to the fact that the $\mathrm{pH}$ of the sol ( $\mathrm{pH} 2)$ is very near to the isoelectric point of the particles, so that the double layer around the particles is insufficient to provide the repulsive electrostatic forces necessary to overcome the gravitational forces and the attractive van der Waals forces. Stability over time was improved by adding TPAH, whose major role is to shift up the $\mathrm{pH}$ far away from the isoelectric point of the particles. Hence, the suspension of glass particles with the lowest TPAH addition (10\% wt\%) settles down instantaneously, so that the measurements were not reliable. Suspensions with 20 and $30 \mathrm{wt} \%$ TPAH give a significant thixotropic cycle, thus suggesting that the suspension is continuously evolving over time. As TPAH content increases to 40 and $50 \mathrm{wt} \%$ the thixotropy and the viscosity decrease, this demonstrating that stability is improved.

The same evolution was observed for the glassceramic suspensions in which the viscosity decreases for increasing TPAH contents, also excepting that with $10 \mathrm{wt} \%$, unable to stabilize the suspension. The glassceramic suspensions showed lower viscosities than the glass suspensions and no thixotropic cycle was observed. This is likely a consequence of the smaller particle size of the glass-ceramic particles.

In both cases, the suspensions that exhibited better stability over time were those in which $50 \mathrm{wt} \%$ (on a dry solid basis) of TPAH was added, the $\mathrm{pH}$ being 6-7. Two different stabilising mechanisms could be responsible for the stability of TPAH-containing suspensions. First, TPAH acts as a strong base and shifts the $\mathrm{pH}$ far away from the isoelectric point of the particles. With this consideration, the isoelectric point of the particles does not change but at pH 6 it should be expected that the particles are negatively charged and should migrate toward the anode in an electrophoresis test. A second approach should be made taking into account that TPAH is a quaternary ammonium and behaves as a cationic surfactant. In this case, stability could be related to the adsorption of the $\mathrm{TPA}^{+}$cations on the particle surface. As a result, the specific adsorption of a cation on the particle should make the isoelectric point shift up and the particles would maintain a positive surface charge of $\mathrm{pH} 6$.

With these considerations in mind, electrophoresis experiments were carried out in order to determine the surface charge of the particles in the presence of TPAH. Cathodic deposition occurred for suspensions without TPAH and with additions of up to $30 \%$, where a $\mathrm{pH}$ value of 6 is reached. Further additions of TPAH reverted the sign of the migration so that deposition became anodic. That means, the isoelectric point occurs at a $\mathrm{pH}$ around 6 , much higher than expected for silica sols and even for wollastonite and other similar silicates, whose isoelectric point is typically lower than $\mathrm{pH} 2$.

Hence, stability is not simply provided by electrostatic repulsion of the double layers, but steric forces related to the specific adsorption of the TPA ${ }^{+}$ions may be also present. In addition, the particle size is too large 


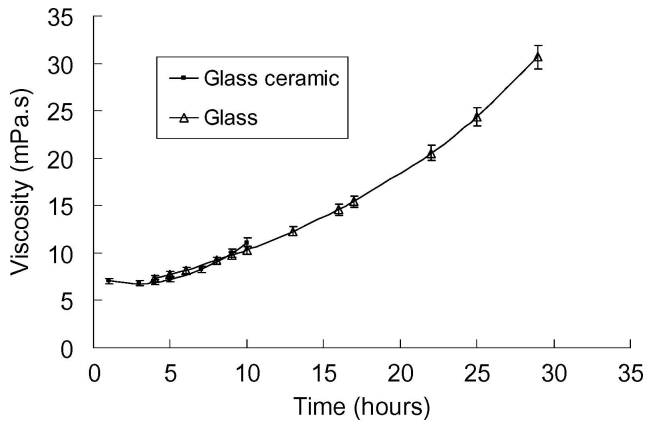

Figure 5. Evolution of viscosity with time of the suspensions with $10 \mathrm{wt} \%$ of glass and glass-ceramic particles with $50 \mathrm{wt} \%$ of TPAH (referred to dry solids).

to allow simple electrostatic stabilization. The better stability of TPAH-containing suspensions supports that some steric contribution might also be present.

The evolution of viscosity with elapsed time was studied for the most stable suspensions (i.e. those with additions of TPAH of $50 \mathrm{wt} \%$ with regard to solids content). These variations are shown in Fig. 5 for both particulate systems. Both suspensions are quite stable for at least $6 \mathrm{~h}$ after preparation, where viscosity maintains a constant value of $7 \mathrm{mPa} . \mathrm{s}$ and only after $9 \mathrm{~h}$ do the viscosities start to increase. Solid-liquid separation started after $29 \mathrm{~h}$ in the suspension of glass particles and after $12 \mathrm{~h}$ in that of glass-ceramic particles; afterwards, the suspensions gelified. Thus, the stability of the suspensions is enough to prepare the films without risk of particle sedimentation and heterogeneous deposition during the dip-coating process which takes only five minutes to complete in each sample.

\subsection{Characterization of the Coatings}

The coatings obtained from the suspensions containing either glass or glass ceramic particles are constituted by a glassy matrix with a secondary phase of particles with a concentration of 10-15 vol\%, depending on the withdrawal rate and the evaporation rate. The embedded particles present a bimodal size distribution, the median values occurring at 20 and $7 \mu \mathrm{m}$. They appear separated from each other, showing uniform distribution along the entire coatings, which demonstrates the stability and homogeneity of the precursor suspension. The critical thickness is around $2 \mu \mathrm{m}$ in both types of coatings. Some defects (like circular cracks around the biggest particles) are observed for thicker coatings, probably due to the difference between the expansion coefficients of the dispersed particles and the glass matrix, and to the presence of fairly big particles, so that the thickness on the border of the particles is much larger than the critical thickness.

Figures 6 and 7 show the microstructure of coatings of $1.5 \mu \mathrm{m}$ in thickness, prepared with the glass and the glass-ceramic particles, respectively. As observed, the layers are free of cracks and big pores, which would

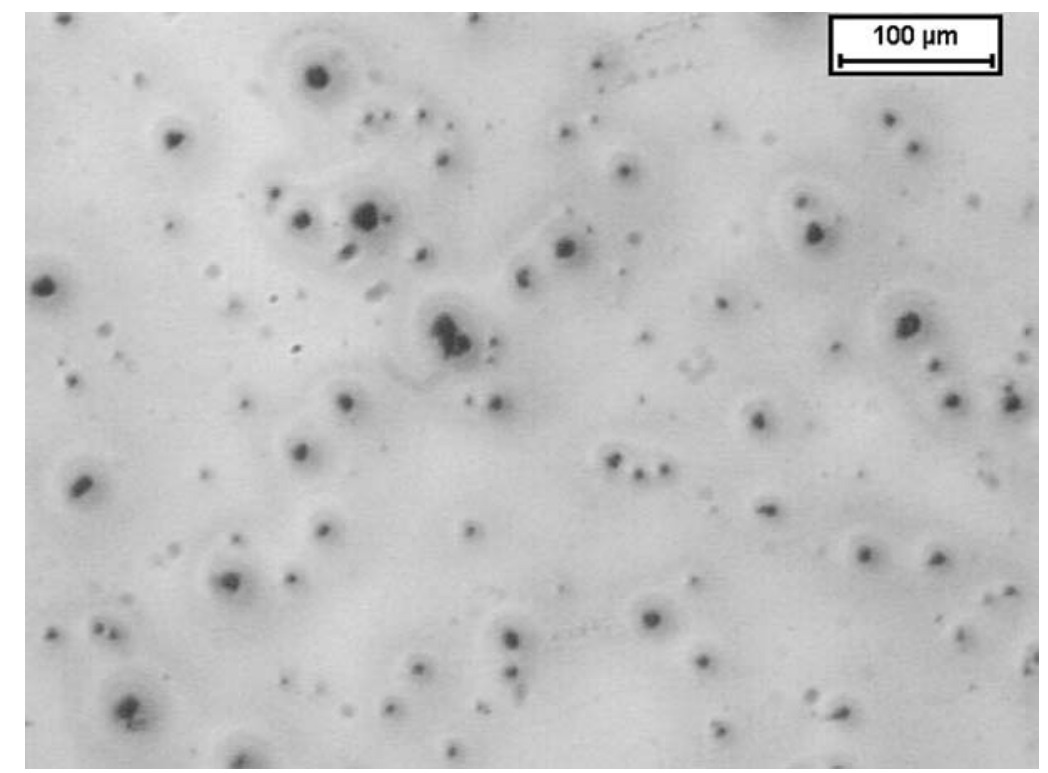

Figure 6. Appearance of the coating with glass particles (X 200). The black points correspond to the glass particles. 


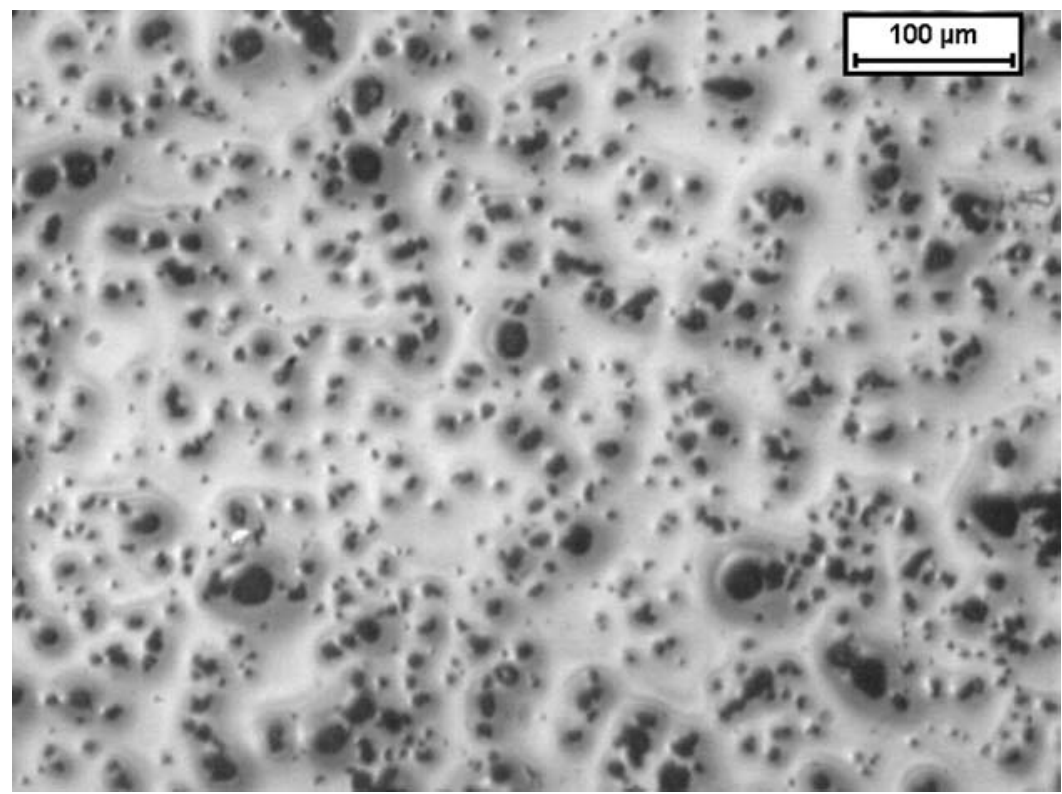

Figure 7. Appearance of the coating with glass ceramic-particles (X 200). The black points correspond to the glass-ceramic particles.

allow an electrolyte to reach the substrate and decrease the corrosion resistance.

\section{Conclusions}

Stable particulate suspensions have been prepared in a hybrid $\mathrm{SiO}_{2}$ sol by adding $10 \mathrm{wt} \%$ of either glass or glass-ceramic particles. The starting $\mathrm{pH}$ of the sol is 12 , close to the isoelectric point of silica. Stability of the suspensions was reached by adding up to $50 \mathrm{wt} \%$ (on a dry solids basis) of tetrapropylammonium hydroxide as a dispersing aid, the final $\mathrm{pH}$ being $6-7$. At these $\mathrm{pH}$ conditions the sol maintains stability, whereas the particles are effectively dispersed through combined stabilisation mechanisms involving the adsorption of the cationic surfactant and the corresponding shift up of the isoelectric point that enhances electrostatic repulsion. The suspensions maintain stability without sedimentation for several hours, thus allowing the preparation of homogeneous coatings without cracks or big pores consisting on a glassy matrix with 10-15 vol\% of glass or glass-ceramic particles as a secondary phase, the last showing a homogeneous distribution across the coating.

\section{Acknowledgments}

This work has been supported by CYTED, Project VIII-9 and Network VIII-E, DINAIN project
DI00C272 and DIME project 30802494 of the Universidad Nacional de Colombia, and CICYT Spanish project MAT2000-0952-C02-01.

\section{References}

1. P.C. Innocenzi, M. Guglielmi, M. Gobbin, and P. Colombo. J. Europ. Ceram. Soc. 10, 431-436 (1992).

2. M.A. Villegas, M. Aparicio, and A. Durán. J. Non-Cryst. Solids 218, 146-150 (1997).

3. T.P. Chou, C. Chandrasekaran, S.J. Limmer, S. Seraji, Y. Wu, M.J. Forbess, C. Nguyen, and G.Z. Cao, J. Non-Cryst Solids 290, 153-162 (2001).

4. D. Chen, Solar Energy Materials \& Solar Cells 68, 313-336 (2001).

5. E. Milella, F. Consentino, A. Licciulli, and C. Massaro, Biomaterials 22, 1425-1431 (2001).

6. P. Galliano, J.J. Damborenea, M.J. Pascual, and A. Durán, J. Sol-Gel Sci. Techn. 13, 723-727 (1998).

7. P. Li, K. de Groot, and T. Kokubo, J. Sol-Gel Sci. Techn. 7, 27-34 (1996).

8. O. de Sanctis, L. Gomez, N. Pellegri, and C. Parodi, J. NonCryst. Solids 121, 338-343 (1990).

9. J. Gallardo, P. Galliano, R. Moreno, and A. Durán, J. Sol-Gel Sci. Techn. 19, 107-111 (2000).

10. T. Kokubo, H. Kushitani, C. Ohtsuki, S. Sakka, and T. Yamamuro, J. Mat. Sci.: Mat Med. 3, 79-83 (1992).

11. Ch. Ohtsuki, T. Kokubo, and T. Yamamuro, J. Non Cryst. Sol. 143, 84-92 (1992).

12. I. Rehman, L.L. Hench, W. Bonfield, and R. Smith, Biomaterials 15(10), 865-870 (1994). 